\title{
Broadband all-optical plane-wave ultrasound imaging system based on a Fabry-Perot scanner
}

\author{
Khoa Pham, Sacha Noimark, Nam Huynh, Edward Zhang, Filip Kuklis, Jiri Jaros, Adrien Desjardins, \\ Ben Cox, Paul Beard
}

\begin{abstract}
A broadband all-optical plane-wave ultrasound imaging system for high-resolution 3D imaging of biological tissues is presented. The system is based on a planar Fabry-Perot (FP) scanner for ultrasound detection and the photoacoustic generation of ultrasound in a Carbon-NanotubePolydimethylsiloxane (CNT-PDMS) composite film. The FP sensor head was coated with the CNT-PDMS film which acts as an ultrasound transmitting layer for pulse-echo imaging. Exciting the CNT-PDMS coating with nanosecond laser pulses generated monopolar plane-wave ultrasound pulses with MPa-range peak pressures, and a -6dB bandwidth of $22 \mathrm{MHz}$, that were transmitted into the target. The resulting scattered acoustic field was detected across a $15 \mathrm{~mm} \times 15 \mathrm{~mm}$ scan area with a step size of $100 \mu \mathrm{m}$ and an optically defined element size of $64 \mu \mathrm{m}$. The $-3 \mathrm{~dB}$ bandwidth of the sensor was $30 \mathrm{MHz}$. A 3D image of the scatterer distribution was then recovered using a $k$-space reconstruction algorithm. To obtain a measure of spatial resolution, the instrument line-spread function (LSF) was measured as a function of position. At the centre of the scan area the depth dependent lateral LSF ranged
\end{abstract}

Manuscript received June 24, 2020; accepted September 28, 2020. This work was supported in part by the Engineering and Physical Sciences Research Council (EPSRC)-funded University College London (UCL) Centre for Doctoral Training in Medical Imaging under Grant EP/L016478/1, the Department of Health's National Institute for Health Research (NIHR)-funded Biomedical Research Centre at University College London Hospitals, the EPSRC under Grant EP/S001506/1, the European Research Council under Advanced Grant 741149, the Wellcome/EPSRC Centre for Surgical and Interventional Sciences under Grant NS/A000050/1, the European Union's Horizon 2020 research program H2020 ICT 2016-2017 under Grant 732411, and the Ministry of Education, Youth and Sports of the Czech Republic from the National Programme of Sustainability (NPU II) through project IT4Innovations Excellence in Science under Grant LQ1602. (Corresponding author: Khoa Pham.)

Khoa Pham and Ben Cox are with the Department of Medical Physics and Biomedical Engineering, University College London, London WC1E 6BT, U.K. (email: khoa.pham.16@ucl.ac.uk).

Sacha Noimark is with the Department of Medical Physics and Biomedical Engineering, University College London, London WC1E 6BT, U.K., also with the Wellcome/EPSRC Centre for Surgical and Interventional Sciences, University College London, London W1W 7TY, U.K., and also with the Materials Chemistry Research Centre, Department of Chemistry, University College London, London WC1H 0AJ, U.K.

Nam Huynh, Edward Zhang, Adrien Desjardins and Paul Beard are with the Department of Medical Physics and Biomedical Engineering, University College London, London WC1E 6BT, U.K., and also with the Wellcome/EPSRC Centre for Surgical and Interventional Sciences, University College London, London W1W 7TY, U.K.

Filip Kuklis and Jiri Jaros are with the Centre of Excellence IT4Innovations, Faculty of Information Technology, Brno University of Technology, 61200 Brno, Czech Republic.

This article has supplementary downloadable material available at https://doi.org/10.1109/TUFFC.2020.3028749, provided by the authors.

Digital Object Identifier 10.1109/TUFFC.2020.3028749 from 46 to $65 \mu \mathrm{m}$ for depths between 1 and $12 \mathrm{~mm}$. The vertical LSF was independent of position and measured to be $44 \mu \mathrm{m}$ over the entire field of view. To demonstrate the ability of the system to provide high-resolution 3D images, phantoms with well-defined scattering structures of arbitrary geometry were imaged. To demonstrate its suitability for imaging biological tissues, phantoms with similar impedance mismatches, sound speed and scattering properties to those present in tissue, and ex vivo tissue samples were imaged. Compared to conventional piezoelectric based ultrasound scanners this approach offers the potential for improved image quality and higher resolution for superficial tissue imaging. Since the FP scanner is capable of high-resolution 3D photoacoustic imaging of in vivo biological tissues, the system could ultimately be developed into an instrument for dual-mode all-optical ultrasound and photoacoustic imaging.

Index Terms-Acoustic sensors, Fabry-Perot interferometer, plane wave, ultrasonic imaging, ultrasonic transducers.

\section{INTRODUCTION}

Conventional biomedical ultrasound scanners often operate over a relatively low narrowband frequency range and have large detection elements limited by the piezoelectric material, both limiting the image resolution and fidelity. For high-resolution tissue imaging applications, these limitations can potentially be addressed with an all-optical ultrasound system that is broadband in both transmission and detection, and provides a high density of acoustic elements.

Optical ultrasound generation can be achieved by photoacoustically generating ultrasound in absorbing materials. Using short laser pulses, broadband high-frequency ultrasound waves can be generated in a range of materials [1]. Polydimethylsiloxane (PDMS) composite absorbers have been shown to exhibit a high thermoelastic conversion efficiency and provide broadband acoustic generation with bandwidths in the tens of MHz range [2], [3]. The optical generation of broadband plane-wave ultrasound for imaging has been demonstrated using a black polyethylene film [4], and more recently using a carbon-black doped PDMS film [5]. Both systems, however, relied on narrowband piezoelectric transducers for detection and thus were not able to take full advantage of the high frequencies generated. The use of a linear array also meant these systems were only capable of 2D imaging. Broadband optical generation of ultrasound has been combined with broadband detection using Fabry-Perot (FP) etalon-based 
ultrasound detectors [6], [7]. The sensors used, however, provided relatively low acoustic sensitivity. As a result, these systems were only able to produce images of strongly scattering wire targets with significant signal averaging. A system that is broadband in both ultrasound generation and detection with high acoustic sensitivity was demonstrated by placing a Carbon-Nanotube(CNT)-PDMS composite film, providing MPa-range peak pressures [8], and a FP sensor on the end of adjacent optical fibres [9]. By mechanically translating the probe, a virtual array of ultrasound transmit-receive elements was created, with which high-resolution and high-fidelity images of biological tissue were obtained.

In this work we present an all-optical plane-wave 3D ultrasound imaging system based on the planar FP scanner [10] for ultrasound detection and a CNT-PDMS composite coating for photoacoustic ultrasound generation. The planar FP scanner simultaneously provides high sensitivity, a high density of small (sub-100 $\mu \mathrm{m}$ ) acoustic elements over a centimetre-scale area, and a broad bandwidth extending to several tens of $\mathrm{MHz}$. These features make it a highly suitable all-optical ultrasound detection system for high-resolution 3D ultrasound imaging of biological tissues. It has also been shown to provide highresolution $3 \mathrm{D}$ in vivo photoacoustic images of biological tissues up to depths of approximately $10 \mathrm{~mm}$ [11], [12]. Modifying the planar FP scanner to be able to also perform pulse-echo ultrasound imaging as described in the current study offers the future prospect of realising a 3D dual-mode photoacoustic and ultrasound imaging system. Such a system has the potential to add complementary morphological ultrasound contrast to photoacoustic vascular images and thus extend its clinical applicability.

In this paper, a description of the system and its evaluation is provided. Section II describes the ultrasound generation and detection mechanisms, and the image reconstruction method. In Section III, we describe the system's ultrasound generation and detection performance, and the spatial resolution it can provide. Finally, ultrasound images obtained by the system of scattering phantoms, tissue mimicking phantoms and ex vivo tissues are presented in Section IV.

\section{OPTICAL ULTRASOUND SCANNER}

The all-optical plane-wave ultrasound scanner developed in this work is based on the planar FP scanner [10]. It comprises an acoustic sensor based on a FP polymer film etalon that is interrogated by a focused laser beam which enables acoustically-induced changes in the etalon thickness to be detected, see Fig. 1. The FP sensor head was coated with a CNT-PDMS composite layer that acted as an ultrasound transmitter coating. The coating was excited with nanosecond laser pulses delivered through the back of the FP sensor head. This generated plane-wave ultrasound pulses which propagated into the imaging target. The acoustic field scattered from the target was then mapped in 2D by the FP scanner, from which a 3D image of the scatterer distribution was recovered using a $k$ space reconstruction algorithm. This section lays out the details of the ultrasound generation, detection and image reconstruction.

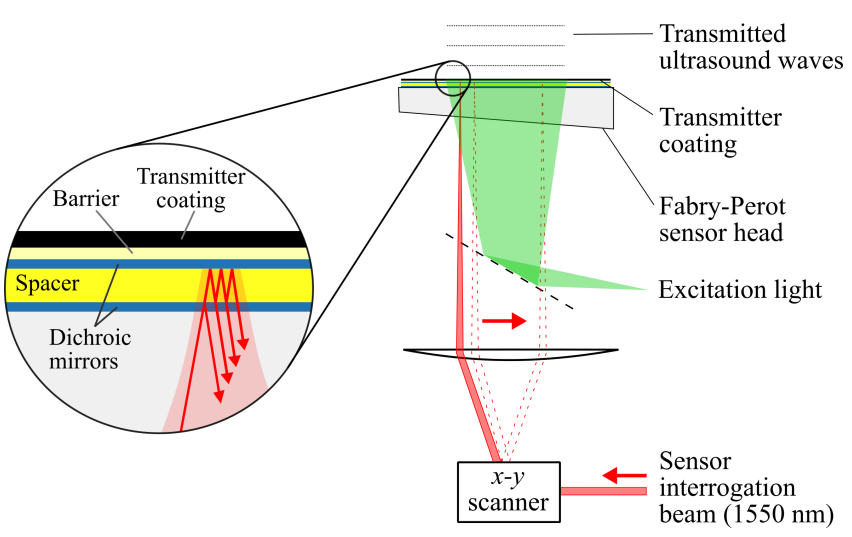

Fig. 1. Schematic of all-optical ultrasound imaging system, based on laser-generated plane-wave ultrasound waves produced by the absorption of laser pulses in a CNT-PDMS composite transmitter coating, and detection of scattered signals with a planar FP scanner.

\section{A. Optical ultrasound generation}

CNT-loaded PDMS films were prepared using the method described by Noimark et al [8]. Multi-walled CNTs were functionalised for uniform dispersion in xylene $(1.8 \mathrm{~mL})$, and manually mixed with PDMS (1 g, MED-1000, Polymer Systems Technology, U.K.). Using doctor-blading methods, the solution was applied onto a polyimide film and cured at room temperature for 24 hours, before removing from the polyimide film to give a thin CNT-PDMS film with an approximate thickness of 60-100 $\mu \mathrm{m}$. In order to coat the FP sensor head, the CNT-PDMS film was soaked in ethanol, which made it slightly swell up. After the film was placed on top of the FP sensor head, the ethanol evaporated and caused the film to shrink tightly to the FP sensor surface. Plane-wave ultrasound pulses were generated by illuminating the CNT-PDMS coating with $7 \mathrm{~ns}$ laser pulses from a fibre-coupled $1064 \mathrm{~nm}$ Q-switched Nd:YAG excitation laser (Quantel Laser, Ultra 50) with a pulse energy of $50 \mathrm{~mJ}$, a beam diameter of approximately $20 \mathrm{~mm}$, and a pulse repetition frequency of $20 \mathrm{~Hz}$.

The CNT-PDMS film exhibits low frequency dependent attenuation over the majority of the frequency range relevant to the current study; e.g. a $100 \mu \mathrm{m}$ film attenuates frequencies up to $22 \mathrm{MHz}$ by less than $1.7 \mathrm{~dB}$ [13]. The attenuation of the photoacoustic wave as it propagates through the CNT-PDMS film is thus negligible.

\section{B. Optical ultrasound detection}

The FP sensor consists of two highly reflecting dichroic mirrors separated by a $20 \mu \mathrm{m}$ spacer made of Parylene-C [14] thus forming a polymer etalon, see Fig. 1. A barrier layer of 2.5 $\mu \mathrm{m}$ thick Parylene-C separated the etalon from the transmitter coating. Incident acoustic waves modulate the etalon optical thickness resulting in a corresponding modulation of its reflectivity. The latter is read-out using a continuous wave interrogation laser beam (Yenista Optics, Tunics T100s-HP) focused on a point in the plane of the sensor. The spot size of the interrogation laser was $64 \mu \mathrm{m}$, which defines the element size of the acoustic detector to a first approximation [15]. To achieve optimum sensitivity, the interrogation laser was tuned 
to the wavelength corresponding to the maximum slope of the interferometer transfer function (ITF), hereafter referred to as the bias wavelength [10]. The bias wavelength was selected after the CNT-PDMS coating had been irradiated by the pulsed laser excitation for approximately 3 minutes. This ensured that the sensor attained the same thermal steady state that exists during image acquisition which is necessary for an accurate bias wavelength estimation. The light reflected from the FP sensor is directed via a single-mode fibre-optic circulator (Thorlabs, 6015-3-APC) on to an InGaAs photodiode-amplifier unit (Hamamatsu, G9801-22, -3dB bandwidth $200 \mathrm{MHz}$ ) connected to a $250 \mathrm{MS} / \mathrm{s}$ digitizer (National Instruments, PCI-5114) with a $125 \mathrm{MHz}$ analogue bandwidth, and a $50 \mathrm{kHz}$ high-pass filter. Acoustic pressure time series were recorded at 22,500 spatial points across the sensor by using a precision $x-y$ galvanometerbased scanner (Cambridge Technology, 623XH) to scan the interrogation beam over a $15 \mathrm{~mm} \times 15 \mathrm{~mm}$ area with a step size of $100 \mu \mathrm{m}$. The acquisition time for one scan was approximately $20 \mathrm{~min}$.

\section{Image reconstruction and processing}

The inverse problem for pulse-echo plane-wave ultrasound imaging has previously been solved by Norton and Linzer [16]. The reconstruction algorithm used in this paper follows the work of Cheng and Lu [17], and is based on three main principles: 1) the imaging object is assumed to be a distribution of weak scatterers $a(x, y, z)$, i.e. multiple scattering is neglected; 2) any acoustic field can be written as a sum of propagating plane waves; and 3) any propagating plane wave can be described uniquely in terms of any three of $\left(\omega, k_{x}, k_{y}, k_{z}\right)$, where $\omega$ is frequency, and $k_{x}, k_{y}, k_{z}$ are the wavenumbers in the $x, y, z$ directions, since they are linked by the dispersion relation $(\omega / c)^{2}=k_{x}^{2}+k_{y}^{2}+k_{z}^{2}$, where $c$ is the speed of sound. Exploiting these principles, the acoustic pressure measured on a plane, $p(x, y, t)$, can be Fouriertransformed to provide $P\left(k_{x}, k_{y}, \omega\right)$, and then mapped to $A\left(k_{x}, k_{y}, k_{z}^{\prime}\right)$ via the relation $k_{z}^{\prime}=\omega / c+k_{z}$, where $\omega / c$ is the additional phase due to the time $(z / c)$ it takes the transmitted plane wave to arrive at scatterers at depth $z$. Finally, by computing the inverse Fourier-transform of $A\left(k_{x}, k_{y}, k_{z}^{\prime}\right)$ the $3 \mathrm{D}$ scattering distribution $a(x, y, z)$ is obtained.

Before reconstruction, the measured pressure time series were filtered with a low-pass Butterworth filter with a $-3 \mathrm{~dB}$ cutoff frequency of $30 \mathrm{MHz}$ to remove high frequency noise beyond the acoustic detection bandwidth. The image was then reconstructed onto a $17 \mathrm{~mm} \times 17 \mathrm{~mm}$ grid with $50 \mu \mathrm{m}$ spacing in $x-y$. The voxel size in depth $z$ is given by the temporal sampling interval used multiplied by a factor of $c / 2$. Envelope detection based on a Hilbert transform was performed on the reconstructed image, and time gain compensation was applied if necessary. The images were interpolated to a $25 \mu \mathrm{m} \times 25 \mu \mathrm{m}$ $\times 10 \mu \mathrm{m}$ spacing in $x-y-z$ using Fourier interpolation. Images are presented as maximum intensity projections (MaxIP) or mean intensity projections (MeanIP) through the whole 3D image volume or through slices of the volume. The time for reconstruction and processing using an Intel Core i7-7700K

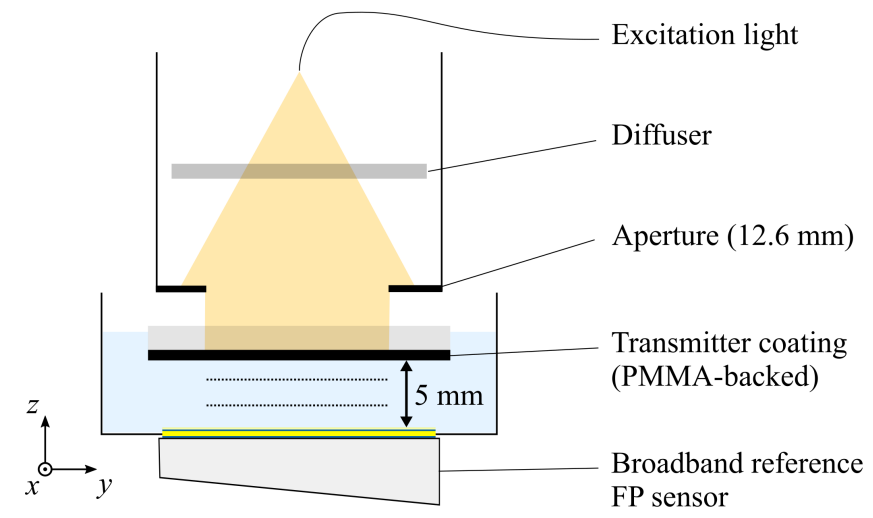

Fig. 2. Set-up for characterizing ultrasound pulses generated by the absorption of laser pulses in a CNT-PDMS composite transmitter coating and propagated through $5 \mathrm{~mm}$ of water, and mapped using a broadband all-hard-dielectric reference FP sensor.

CPU@4.20 GHz and 32 GB RAM was approximately 30 s.

\section{SYSTEM CHARACTERISATION}

\section{A. Mapping of optically generated ultrasound}

The optical ultrasound generation from a photoacoustically excited CNT-PDMS film was characterized in terms of its peak pressure, wave front planarity and bandwidth using the set-up shown in Fig. 2. The output of the excitation laser fibre was passed through a diffuser and an aperture of $12.6 \mathrm{~mm}$ diameter to homogenise its fluence across a known area $\left(22 \pm 2 \mathrm{~mJ} / \mathrm{cm}^{2}\right)$. Note that the diffuser and aperture were only used in this experimental arrangement, not the set-up used for imaging experiments, which is shown in Fig. 1. This excitation beam was delivered to a CNT-PDMS film deposited on a polymethylmethacrylate (PMMA) slab to photoacoustically generate ultrasound. The reference FP sensor used to map the field had an all-hard-dielectric spacer layer and provided a theoretical thickness-mode limited bandwidth in excess of 1 $\mathrm{GHz}$, and a constant frequency response to within $1 \mathrm{~dB}$ in the range 1-40 $\mathrm{MHz}$ [18]. The reference FP sensor was positioned parallel to the CNT-PDMS transmitter film and separated by a distance of $d=5 \mathrm{~mm}$ in water. The incident ultrasound wave was mapped over a $14 \mathrm{~mm} \times 14 \mathrm{~mm}$ area, with a step size of $100 \mu \mathrm{m}$, element size of $64 \mu \mathrm{m}$ and temporal sampling interval of $4 \mathrm{~ns}$. The CNT-PDMS film exhibited a generation efficiency of $0.117 \pm 0.015 \mathrm{MPa} \cdot \mathrm{cm}^{2} / \mathrm{mJ}$ for $\mathrm{d}=5 \mathrm{~mm}$. The mean peak pressure across the wave front was $2.54 \pm 0.24 \mathrm{MPa}$, and the wave was planar to within $\lambda / 10$ at $10 \mathrm{MHz}$, Fig. 3 .

In order to determine the bandwidth of the emitted acoustic plane wave, measurements of the pressure time series were made at a single point in the centre of the reference sensor with a temporal sampling interval of $0.8 \mathrm{~ns}$ using a $350 \mathrm{MHz}$ digitizing oscilloscope (Tektronix, 5034D). A measurement was first obtained with a neutral density (ND) filter with an optical density of 1 at $1064 \mathrm{~nm}$ (90\% attenuation) placed between the aperture and the PMMA backing, in order to reduce the laser pulse energy and consequently the peak pressure to ensure linear acoustic propagation through the water. The measurement was then repeated but without the ND filter in 


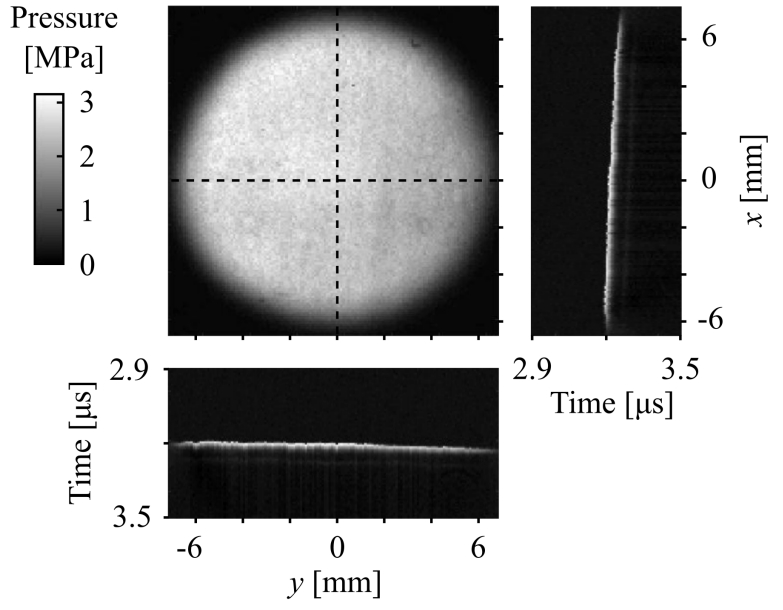

Fig. 3. Map of peak pressure distribution in $x y$ plane, and profiles through the wavefront in $x$ and $y$, produced by CNT-PDMS composite transmitter coating.

place resulting in non-linear propagation. To ensure similar SNR in both cases, 1024 averages were used in the linear case and 12 averages in the non-linear case. Since the former measurement is acquired in the absence of non-linear propagation it provides a measure of the inherent acoustic bandwidth that the coating provides. Monopolar ultrasound pulses with FWHM pulse width of 16 ns were recorded in both cases, see Fig. 4a. For each measurement, the time series was filtered to remove any DC offset, windowed with a $0.4 \mu \mathrm{s}$ Tukey window centred around the peak, before taking the Fourier transform to obtain the frequency spectrum. The linearly propagated pulse had a broadband frequency spectrum with a $-6 \mathrm{~dB}$ bandwidth of $22 \mathrm{MHz}$, Fig. 4b. The non-linearly propagated pulse had a similar $-6 \mathrm{~dB}$ bandwidth but contained more energy at frequencies above $40 \mathrm{MHz}$.

\section{B. Fabry-Perot scanner}

The acoustic bandwidth of the FP sensor used for imaging was measured using a broadband (1-70 MHz) laser-generated ultrasound source [19] and the broadband reference FP sensor described in section III.A. The output of the source was measured at 4 different points on both sensors with a temporal sampling interval $4 \mathrm{~ns}$ and averaged 100 times for the imaging sensor and 1000 times for the reference sensor. Each measurement was filtered to remove any DC offset and windowed with a $0.4 \mu$ s Tukey window centred around the peak, before taking the Fourier transform to obtain the frequency spectrum. Dividing the frequency spectrum of the signal detected by the imaging sensor by that detected by the reference sensor, and averaging over the resulting 16 spectra gave the imaging FP sensor's frequency response, shown in Fig. $4 \mathrm{~b}$ (black dashed line). From this data, the $-3 \mathrm{~dB}$ and $-6 \mathrm{~dB}$ bandwidths of the FP sensor were estimated to be $30 \mathrm{MHz}$ and $34 \mathrm{MHz}$, respectively.

Based on the sensitivity of the sensor described by Zhang et al [10], which was of a similar design, the peak noise equivalent pressure is estimated to be approximately $0.3 \mathrm{kPa}$ over a 20 $\mathrm{MHz}$ measurement bandwidth.
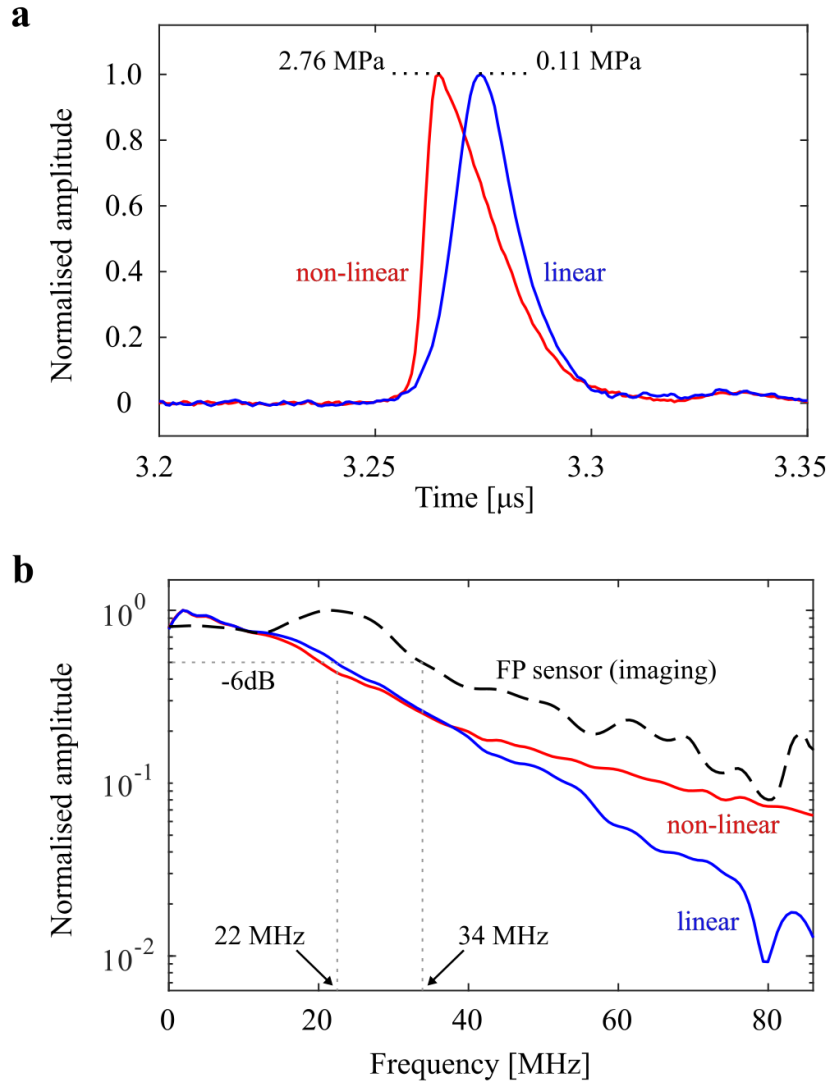

Fig. 4. Characteristics of optically generated ultrasound pulses. (a) Normalised time-domain signals of linearly and non-linearly propagated pulses. (b) Normalised frequency spectra of linearly and non-linearly propagated pulses, and frequency response of FP sensor used for imaging.

\section{Heating effects}

With each laser pulse used to generate an ultrasound wave, heat is deposited in the transmitter coating. This generates a thermal wave that diffuses through the sensor layers, causing thermal expansion of the spacer, and thus a shift in the ITF. This could result in the bias wavelength no longer corresponding to the point of maximum slope on the ITF thus reducing acoustic sensitivity. In this section we assess the effect of the thermal wave on the acoustic sensitivity of the sensor.

The set up for the measurement was the same as for imaging (Fig. 1), with the sensor immersed in a water bath. The distance between the sensor and the water surface was approximately 15 $\mathrm{mm}$. The sensor was interrogated at a single point, and the transmitter coating excited by the pulsed excitation laser for an extended period of several minutes to allow a thermal steady state to be established. During this thermal steady state, the bias wavelength was chosen. By measuring the sensor signal with the $50 \mathrm{kHz}$ high-pass filter on the photodiode-amplifier unit disabled, both thermal and acoustic signals can be monitored simultaneously. Fig. 5a shows the sensor signal over a time scale of two excitation pulses. The sensor was observed to heat up quickly after each excitation pulse, with the deposited heat dissipating between the two excitation pulses. Fig. $5 \mathrm{~b}$ shows the sensor signal over a time scale of $200 \mu$ s around the first excitation pulse, and includes several acoustic signals and the 

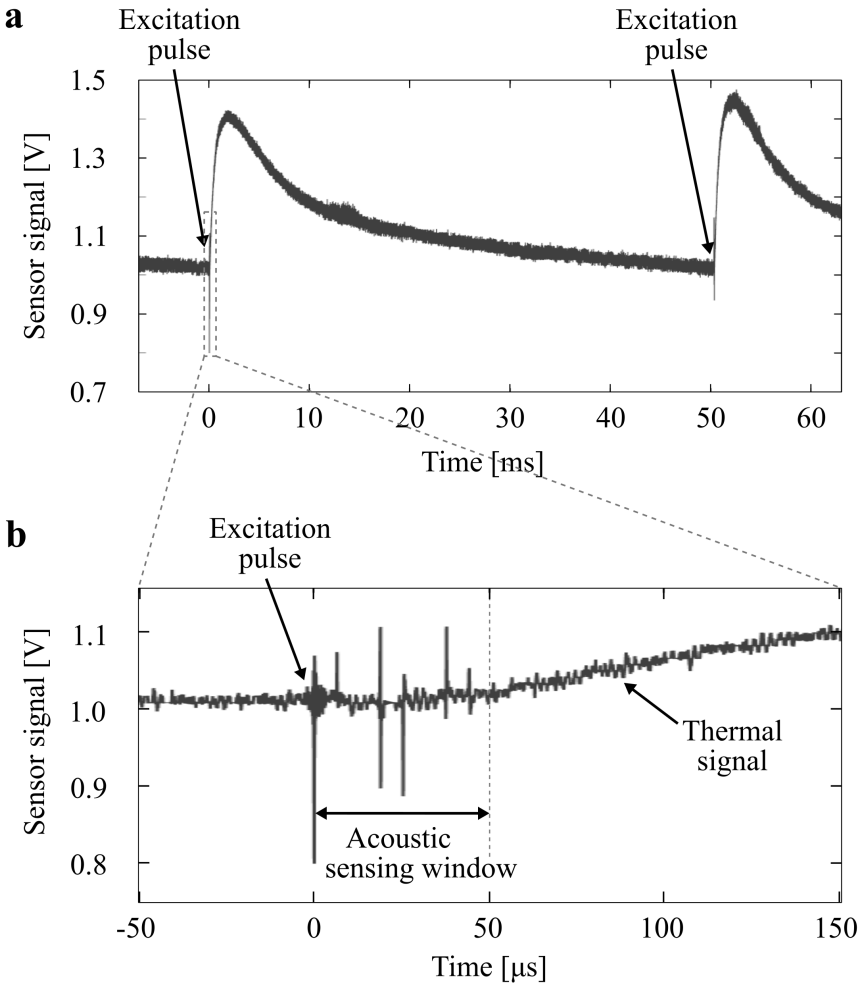

Fig. 5. Time scale of heating effects. Sensor signal shown over a time scale of (a) two excitation pulses and (b) zoomed in on the acoustic sensing window. The acoustic signals seen in the $50 \mu$ s acoustic sensing window in (b) arise from time-delayed reflections of the plane wave emitted by the coating from the interface between the air and the surface of the water layer above the sensor and reflections of the backpropagating wave at the sensor head backing-air interface.

onset of the thermal signal. The arrival of the thermal wave is delayed by about $50 \mu$ s after each excitation laser pulse. This is due to the time it takes for the thermal wave to diffuse through the $2.5 \mu \mathrm{m}$ thick Parylene-C barrier layer, which separates the transmitter coating from the sensor layers (Fig. 1). In this $50-\mu \mathrm{s}$ time window between the excitation and arrival of the thermal wave, acoustic signals can be detected without loss of sensitivity due to thermally induced bias wavelength changes. The equivalent pulse-echo imaging depth of a $50 \mu$ s delay is approximately $37.5 \mathrm{~mm}$. If greater imaging depth is required,

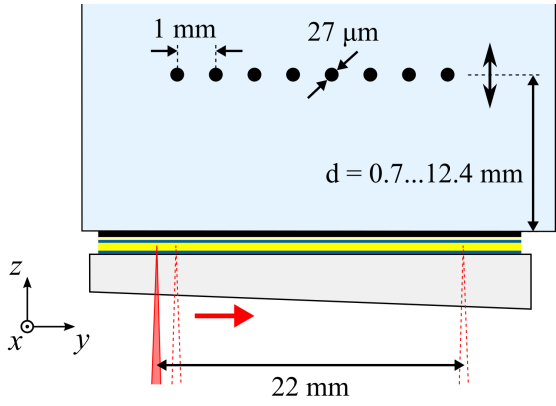

Fig. 6. Set-up used to estimate system line spread function. The phantom comprised a row of $27 \mu \mathrm{m}$ diameter tungsten wires immersed in water, and was positioned at different depths ranging from 0.7 to $12.4 \mathrm{~mm}$. At each depth a 2D image was acquired by scanning the interrogation beam along a line of length $22 \mathrm{~mm}$ in steps of $20 \mu \mathrm{m}$.

the arrival of the thermal wave could be further delayed by increasing the thickness of the barrier coating.

\section{Spatial resolution}

To obtain a measure of the spatial resolution that the system can provide measurements of the instrument line-spread function (LSF) were made. This was achieved by imaging a target comprising a row of strongly scattering tungsten wires (Fisher Scientific, 7440-33-7) immersed in water, see Fig. 6. The wires were $27 \mu \mathrm{m}$ in diameter, spaced by $1 \mathrm{~mm}$ and arranged parallel to each other and the FP sensor. The target was imaged at varying depths from 0.7 to $12.4 \mathrm{~mm}$ in approximately $1 \mathrm{~mm}$ steps. To acquire each image, the sensor interrogation beam was scanned along a line of length $22 \mathrm{~mm}$ in steps of $20 \mu \mathrm{m}$ in the direction perpendicular to the wires, and the acoustic pressure time series recorded at each point with a temporal sampling interval of $4 \mathrm{~ns}$ and without signal averaging. The absorption of the excitation laser pulses in the coating produced heat that diffused away into the surrounding water. This established a temperature gradient in the water bath leading to a slightly varying sound speed as a function of depth. In order to reconstruct the images, a uniform sound speed was assumed, and chosen as $1489 \mathrm{~m} / \mathrm{s}$ to give the highest average resolution for targets within a central $6 \mathrm{~mm} \times 12 \mathrm{~mm}$ window in the $y z$ plane. Each image was reconstructed on a $22 \mathrm{~mm} \times 13$ mm $y-z$ grid with $10 \mu \mathrm{m} \times 6 \mu \mathrm{m}$ spacing, envelope detected,
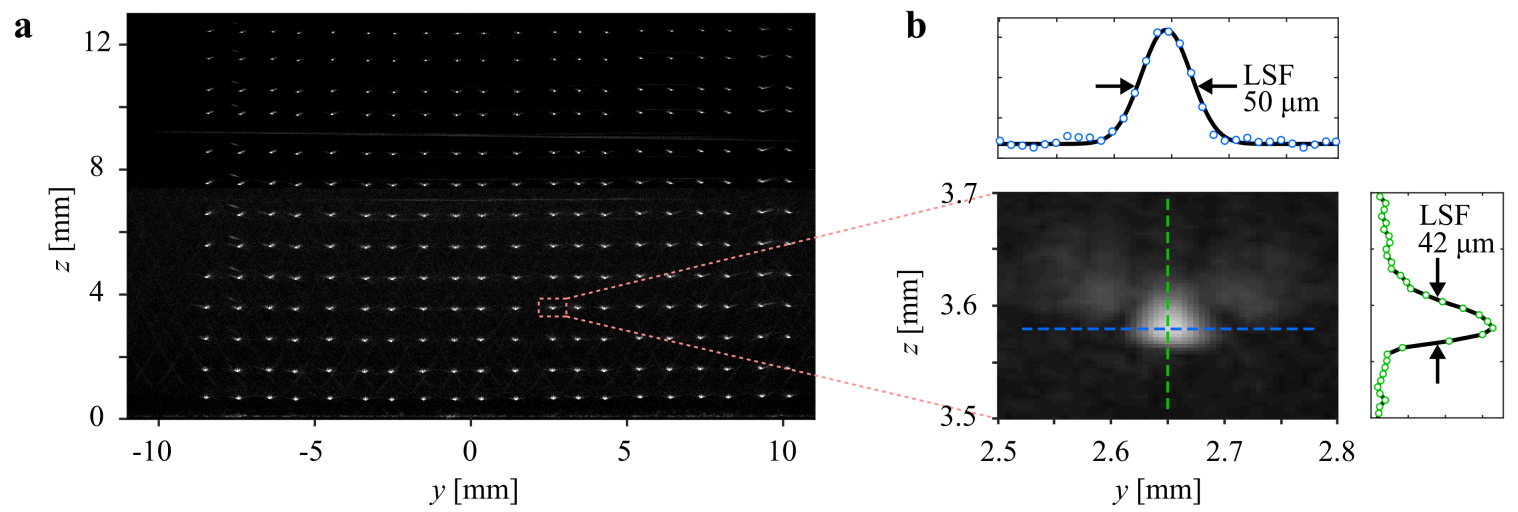

Fig. 7. Estimation of system line spread function. (a) Compound ultrasound image of wires at all depths. (b) Expanded view of single wire. The lateral LSF was determined by fitting a Gaussian function to the profile of the wire image intensity and measuring the FWHM. The axial LSF was determined by linearly interpolating the profile before measuring the FWHM. 


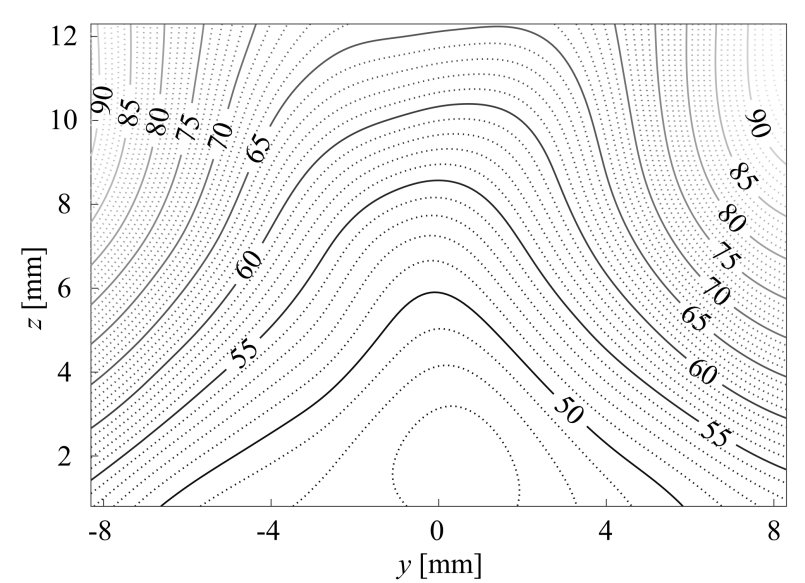

Fig. 8. Contour plot of lateral resolution in $y z$ plane, in units of $\mu \mathrm{m}$.

and interpolated to $5 \mu \mathrm{m}$ in the lateral $y$ direction using Fourier interpolation. Fig. 7a shows the compound image obtained by summing the images of the target acquired at each depth. The lateral and axial LSF were obtained by taking horizontal and vertical profiles through the maximum intensity point of the wire image, and measuring the FWHM, see Fig. 7b. In the lateral direction a Gaussian function was fitted to the profile, while in the axial direction linear interpolation was applied, to determine the FWHM. By doing this for all wires, the spatial dependence of the lateral resolution can be illustrated in a contour plot, see Fig. 8. Given a sound speed uncertainty of 1 $\mathrm{m} / \mathrm{s}$ due to the temperature gradient in the water, the average error in the lateral resolution was estimated to range from 0.5 $\mu \mathrm{m}$ at $1 \mathrm{~mm}$ depth to $6 \mu \mathrm{m}$ at $12 \mathrm{~mm}$ depth. The axial resolution was found to be $44 \pm 3 \mu \mathrm{m}$ across the whole field of view. As expected, the axial resolution showed no significant spatial variation, as it is largely defined by the bandwidth of the generated pulses and the sensor.

\section{IMAGING EXPERIMENTS}

\section{A. Three-dimensional imaging of arbitrarily shaped scatterers}

To demonstrate the ability of the system to provide 3D ultrasound images, phantoms with well-defined scattering structures of arbitrary geometry were imaged.

The first phantom was an optical fibre of $200 \mu \mathrm{m}$ diameter, tied into a knot, positioned approximately $5 \mathrm{~mm}$ away from the sensor and immersed in water, see Fig. 9a. The reconstructed 3D image is presented as MaxIPs in the $x y, x z$ and $z y$ planes, see Fig. 9b. The $z-y$ MaxIP in Fig. 9b demonstrates acoustic shadowing that occurs behind the strongly scattering fibre, where the ultrasound wave was unable to propagate.

The second phantom was a polymer skeleton that resembles a leaf with structures of dimensions in the range of tens to hundreds of $\mu \mathrm{m}$ (Fig. 9c). The average frequency spectrum of the received pressure time series data acquired when imaging the polymer leaf is shown in the appendix. The polymer leaf image was reconstructed and presented in the same way as the knot, see Fig. 9d. For both phantoms, comparison with the photographs shows that the reconstructed image provides an a

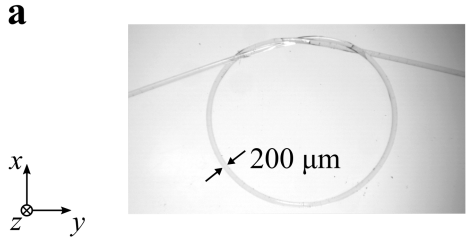

c

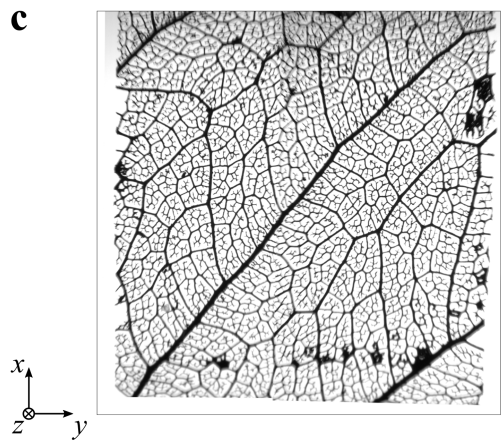

b

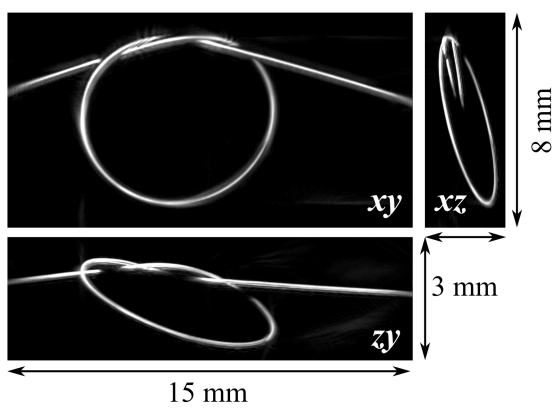

d

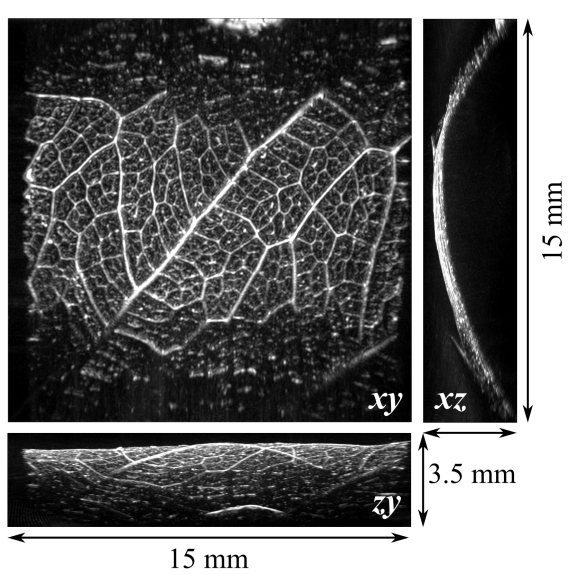

Fig. 9. Ultrasound imaging of arbitrarily shaped scattering structures: (a) Photograph and (b) maximum intensity projections of 3D ultrasound image of optical fibre knot. (c) Photograph and (d) maximum intensity projections of 3D ultrasound image of polymer leaf skeleton. 

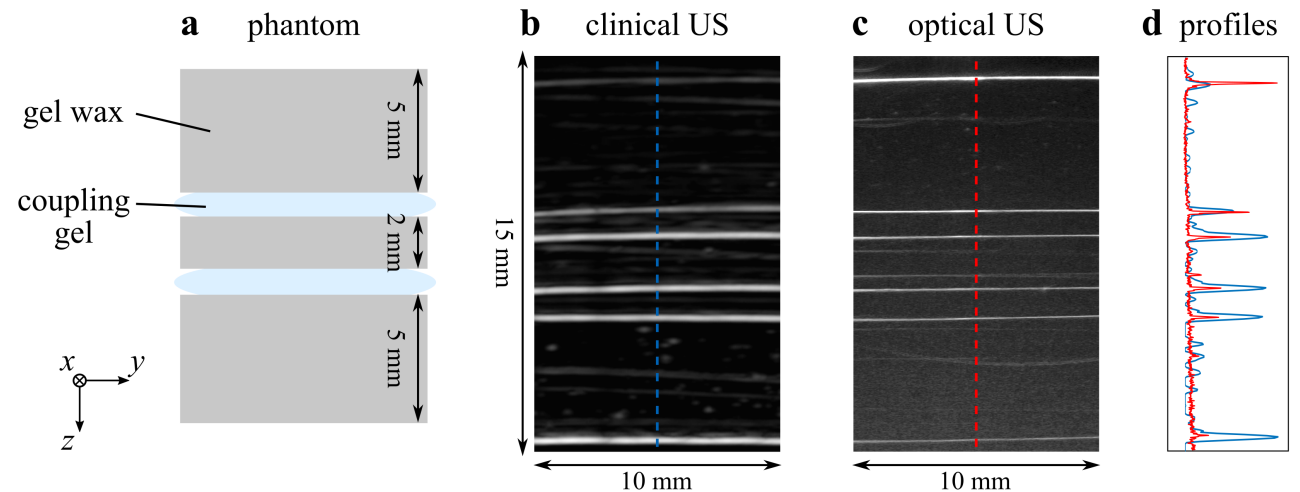

Fig. 10. Ultrasound imaging of layered gel wax-based tissue-mimicking phantom: (a) Schematic of phantom. (b) Clinical ultrasound scanner image, $20 \mathrm{MHz}$ centre frequency. (c) Mean intensity projection through a $2 \mathrm{~mm}$ slice of the 3D all-optical ultrasound image. (d) Comparison of depth profiles through clinical scanner (blue) and FP scanner (red) images.

accurate representation of the scattering structures. As expected, the system was able to resolve structures down to approximately $50 \mu \mathrm{m}$.

\section{B. Imaging of tissue mimicking phantoms}

To demonstrate the feasibility of the system for visualising biological tissues, two tissue-mimicking phantoms (TMM) were imaged with the FP scanner, as well as a clinical ultrasound scanner (Ultrasonix, SonixMDP, in research mode with default settings) for comparison.

The first phantom was designed to have interfaces with acoustic impedance mismatches similar to those present in tissue. It comprised three layers of gel wax [20] (impedance $1.22 \times 10^{6} \mathrm{~kg} \cdot \mathrm{m}^{-2} \cdot \mathrm{s}^{-1}$ ) placed parallel to the sensor surface, see Fig. 10a. The layers were acoustically coupled by ultrasound coupling gel (OptiLube Lubricating Jelly, impedance $1.55 \times 10^{6}$ $\left.\mathrm{kg} \cdot \mathrm{m}^{-2} \cdot \mathrm{s}^{-1}\right)$. This made for an impedance mismatch of $0.33 \times 10^{6}$ $\mathrm{kg} \cdot \mathrm{m}^{-2} \cdot \mathrm{s}^{-1}$ at all boundaries, equivalent to a blood-fat interface $\left(1.66-1.33 \times 10^{6} \mathrm{~kg} \cdot \mathrm{m}^{-2} \cdot \mathrm{s}^{-1}\right)$. The clinical scanner and the FP scanner acquired images from opposite sides of the phantom; the clinical scanner from above and the FP scanner from below. This allowed the phantom to remain stationary and avoid deformation while imaging with both systems. Using the clinical scanner with a high frequency probe (L40-8/12, 20 $\mathrm{MHz}$ centre frequency), a B-mode image of a slice through the phantom was obtained, Fig. 10b. The optical ultrasound image is presented in Fig. 10c as a MeanIP over a slice of thickness 2 $\mathrm{mm}$ through the $3 \mathrm{D}$ image volume. The profiles through the centre of both images show that the FP scanner image provides higher axial resolution than the clinical scanner image, see Fig. 10d. This is expected due to the broader bandwidth of the FP scanner-based system. The relative magnitudes of the peaks in both depth profiles decay in opposite directions along the $z$ axis, since the phantom was imaged from opposite sides by the two scanners. From the depth profile of the FP scanner image (red) the SNR is measured to be 30 at $1 \mathrm{~mm}$ depth, 5 at $10 \mathrm{~mm}$ depth, and 3 at $15 \mathrm{~mm}$ depth.

The second phantom was designed to have sound speeds and scattering coefficients similar to those present in tissue. An agar-based TMM, utilising glycerol for sound speed modification, and orgasol for scattering was made (0.6 g agar, $2.2 \mathrm{~g}$ glycerol, $0.36 \mathrm{~g}$ orgasol, $16.84 \mathrm{~g}$ water). The mixture was poured into a custom-printed mould to make a block of TMM with cylindrical cavities of approximately $1 \mathrm{~mm}$ diameter that were filled with water, see Fig. 11a. Using the clinical scanner with the standard probe (L14-5/38, $14 \mathrm{MHz}$ centre frequency) a B-mode image of a slice through the phantom was obtained, see Fig. 11b. The optical ultrasound image was obtained by incoherent frequency compounding to improve image contrast [21]. To implement this, the detected pressure time-series data was band-pass filtered with centre frequencies of $2,3,4, \ldots, 15$ $\mathrm{MHz}$ and a bandwidth of $10 \mathrm{MHz}$ to produce 14 filtered versions of the pressure time-series data. These were then used to reconstruct 14 images, which were envelope detected before they were summed to obtain the compound image. The compounded optical ultrasound image is presented in Fig. 11c
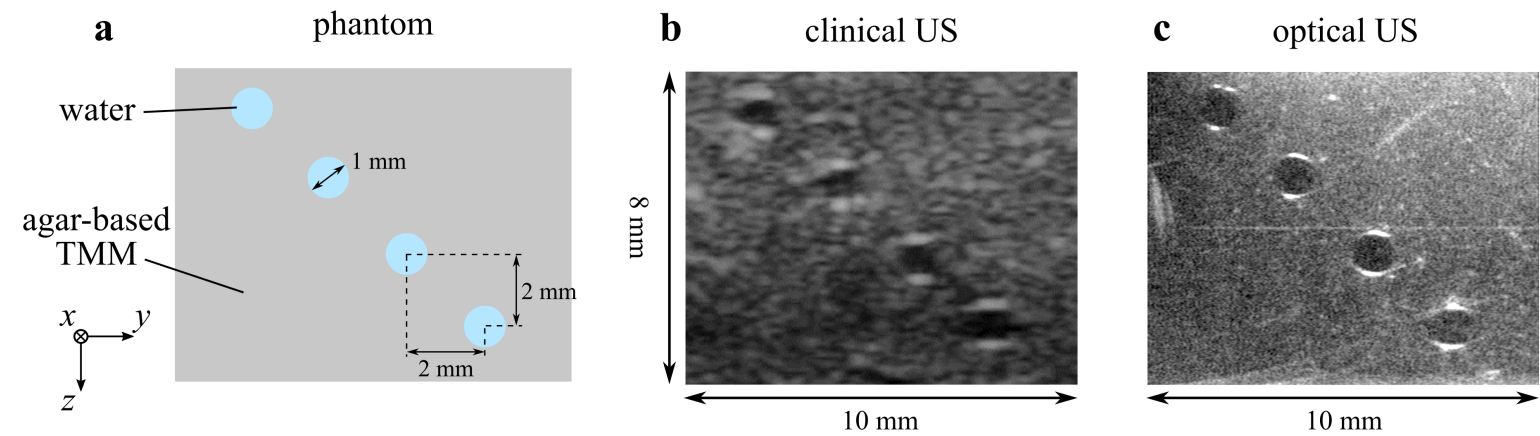

Fig. 11. Ultrasound imaging of agar-based tissue-mimicking phantom. (a) Schematic of phantom. (b) Clinical ultrasound scanner image, 14 $\mathrm{MHz}$ centre frequency. (c) Mean intensity projection through a $2 \mathrm{~mm}$ slice of the 3D all-optical ultrasound image. 
$\mathbf{a}$

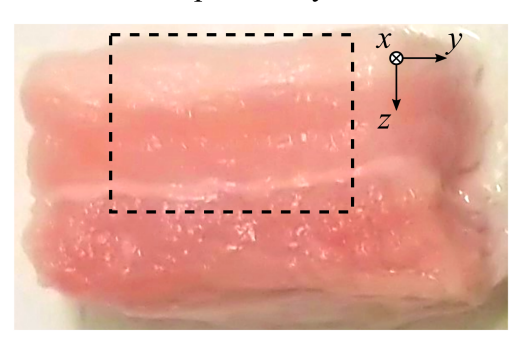

$\mathbf{b}$

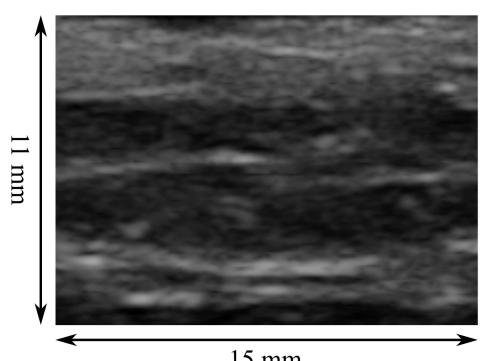

c

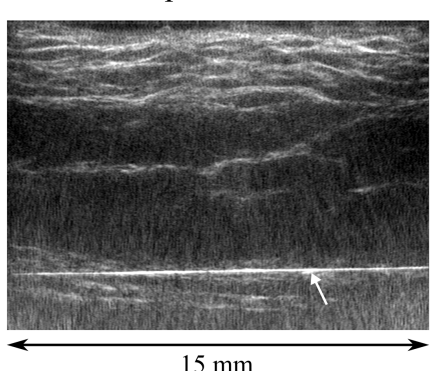

Fig. 12. Ultrasound imaging of ex vivo pork belly. (a) Photograph of pork belly sample showing layers of fat and muscle tissue, and imaging ROI. (b) Clinical ultrasound scanner image, $14 \mathrm{MHz}$ centre frequency. (c) Mean intensity projection through a $2 \mathrm{~mm}$ slice of the 3D all-optical ultrasound image. Line feature marked with an arrow is an image artefact corresponding to the reflection of the back-propagating plane-wave from the back of the sensor head. A fly-through of the 3D optical ultrasound image volume is included as a supplementary AVI video clip, available at http://ieeexplore.ieee.org.

as a MeanIP over a slice of thickness $2 \mathrm{~mm}$ through the 3D image volume. Comparing the two images in Fig. $11 \mathrm{~b}$ and Fig. $11 \mathrm{c}$, the FP scanner image is observed to show the boundaries of the holes with higher resolution and exhibit a finer grain of speckle, both due to the broader generation and detection bandwidth of the FP scanner system.

\section{Ex vivo tissue imaging}

The suitability of the system for imaging soft tissues was evaluated by imaging a piece of pork belly immersed in water, see Fig. 12a. Using the clinical scanner with the standard probe (L14-5/38, $14 \mathrm{MHz}$ centre frequency) a B-mode image of a slice through the tissue was obtained, Fig. 12b. The optical ultrasound image of approximately the same tissue region is presented in Fig. 12c as a MeanIP over a slice of thickness 2 $\mathrm{mm}$ through the $3 \mathrm{D}$ image volume. The images show some structural resemblance, as both exhibit stronger scattering in the superficial fat layer, and a lateral structure within the muscle tissue below. The high resolution of the FP scanner image is illustrated by the representation of the fat layer, which shows striations that are not clearly resolved in the clinical scanner image. These striations are likely to represent the morphology of the fat layer, e.g. the extracellular matrix. The line feature marked with an arrow in the optical ultrasound image is an image artefact corresponding to the reflection of the plane-wave from the back face of the sensor head and could be avoided by increasing the thickness of the sensor backing. The average frequency spectrum of the received pressure time series data is shown in the appendix.

An ex vivo human lymph node (Fig. 13a) immersed in water was also imaged with the FP scanner and the clinical scanner. Fig. 13b shows MaxIPs through the entire 3D image volume, $\mathbf{a}$

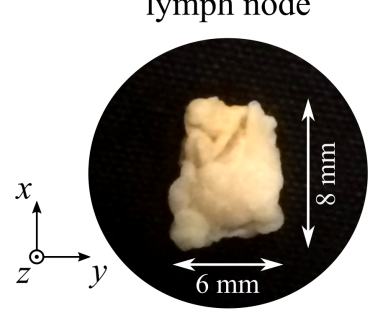

$\mathbf{b}$

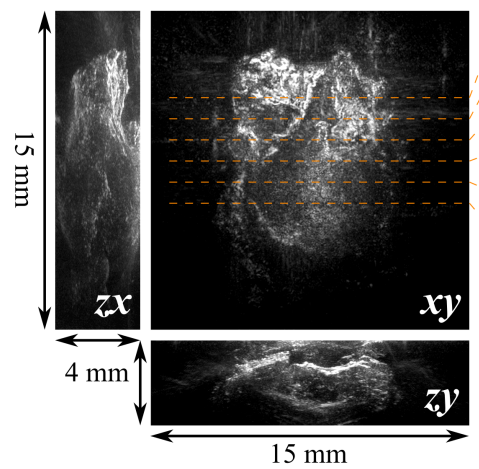

c optical US
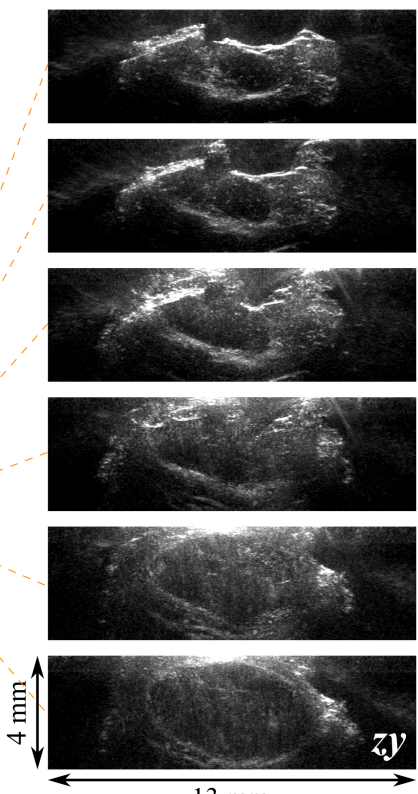

$13 \mathrm{~mm}$ d clinical US
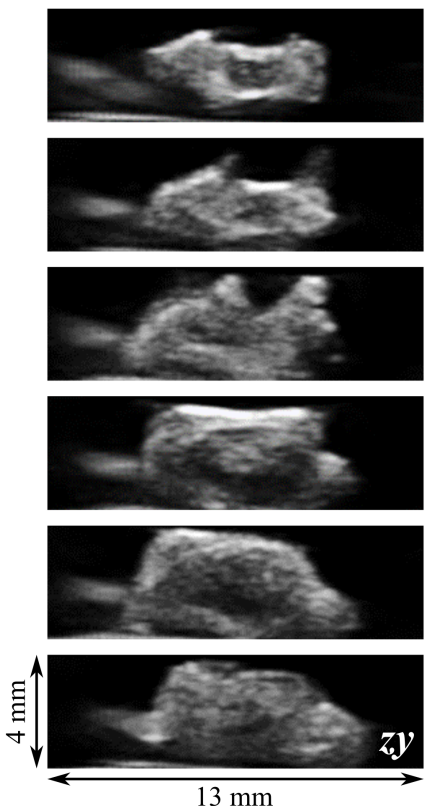

Fig. 13. Ultrasound imaging of ex vivo human lymph node. (a) Photograph of lymph node. (b) Maximum intensity projections through whole volume of the 3D all-optical ultrasound image. (c) Maximum intensity projections through $2 \mathrm{~mm}$ slices of the all-optical ultrasound image in $z y$ plane, and (d) corresponding clinical ultrasound images, $14 \mathrm{MHz}$ centre frequency. A fly-through of the 3D optical ultrasound image volume is included as a supplementary AVI video clip, available at http://ieeexplore.ieee.org. 
while Fig. 13c represents a series of MaxIPs through different $z-y$ slices of $2 \mathrm{~mm}$ thickness. The clinical scanner images of the equivalent $z-y$ slices obtained with the standard probe (L14$5 / 38,14 \mathrm{MHz}$ centre frequency) are shown in Fig. 13d. In the $x-y$ MaxIP of the whole 3D image volume (Fig. 13b), the sharp fold in the top surface of the lymph node is clearly visible. In the $z-y$ slice MaxIPs (Fig. 13c) the boundary between the fat surrounding the lymph node and the water can be seen. Typical for ultrasound images of lymph nodes, a weakly echogenic 'dark void' in the centre of the node is observed in the slice images in Fig. 13c and Fig. 13d.

\section{CONCLUSION}

An all-optical system for pulse-echo plane-wave ultrasound imaging has been demonstrated. The system is based on the planar FP scanner for ultrasound detection. Optical ultrasound generation was achieved by coating the sensor head of the FP scanner with a CNT-PDMS film and exciting it with nanosecond laser pulses to generate plane-wave ultrasound pulses. The generated ultrasound pulses had MPa-range peak pressures, a $-6 \mathrm{~dB}$ bandwidth of $22 \mathrm{MHz}$ and were planar to within $\lambda / 10$ at $10 \mathrm{MHz}$. It has been demonstrated that the system can provide high-resolution high-fidelity 3D ultrasound images of scattering structures, tissue mimicking phantoms and ex vivo tissues.

Compared to conventional ultrasound scanners, the system offers the prospect of improved image quality for superficial tissue imaging. High-resolution imaging is enabled due to the high frequencies the system operates at and the fine spatial sampling of the sensor, while the broadband generation and detection provide spatial fidelity. Another advantage is the 3D imaging capability of the system, as most conventional highfrequency ultrasound scanners form 2D images. In its current state the system is able to provide a penetration depth of up to $10 \mathrm{~mm}$ for in vivo tissue imaging. However, there remains scope to improve its performance in this respect. By modifying the temporal characteristics of the excitation laser (e.g. the pulse width), for example by using a fibre laser [22], the frequency content of the generated ultrasound can be tuned to increase the energy at lower frequencies which are attenuated less and thus penetrate more deeply in tissue. Spatial or angle compounding also has the potential to improve SNR and thus penetration depth and image contrast [23], [24]. Photoacoustic generation of angled plane waves can be achieved by scanning a focused excitation beam across the transmitter coating [25]. A limitation of the system is the long acquisition time due to the low PRF of the excitation laser used, the large number of detection points required for each scan and the sequential nature of the sensor read-out. This can be addressed by using a higher PRF laser and parallelizing the sensor read-out by the use of multiple interrogation beams [26] or widefield illumination and a camera-based sensor array [27]. The bandwidth of the system is currently limited by the laser pulse duration and thicknesses of the FP sensor spacer and the CNT-PDMS film. By reducing all three, there is the potential to increase the bandwidth to 100 $\mathrm{MHz}$ or higher, albeit at the cost of sensitivity and thus image SNR.

Possible clinical applications of this system are highresolution imaging of the skin and superficial lymph nodes. The system can be modified for endoscopic use by placing both the sensor [28] and the transmitter coating [29] at the distal end of a fibre bundle, and delivering the interrogation and excitation light through the bundle. This would have the advantage of a higher element density compared to endoscopic piezoelectric arrays. These modifications would also make the system MRI compatible since separating the galvanometer mirrors from the sensor head makes it completely free of electronics and metallic components. With its broadband generation and detection capability the system could also serve as a research system to explore the effect of different frequency regimes on image characteristics such as contrast, speckle and spatial fidelity in plane-wave ultrasound imaging. Since this all-optical ultrasound imaging system is based on the same FP scanner that has been shown to obtain excellent high-resolution $3 \mathrm{D}$ in vivo photoacoustic images, it could be combined with photoacoustic imaging to obtain co-registered dual mode images. One way to realise such a dual-mode system would be to incorporate a coating that is transparent to wavelengths used for photoacoustic imaging but provides strong absorption at a wavelength used for generating ultrasound [2]. This would enable complementary morphological ultrasound contrast to be added to photoacoustic images of optical absorption which could aid the clinical assessment of superficial tumours, lymph nodes and dermatological conditions.

\section{APPENDIX}

The average frequency spectra of the received pressure time series data acquired when imaging the polymer leaf skeleton phantom and the pork belly tissue sample with the FP scanner are shown in Fig. 14. At frequencies above $5 \mathrm{MHz}$, the polymer leaf skeleton spectrum closely resembles the product of the sensor and source spectra, since the leaf was immersed in water which exhibits low acoustic attenuation. The pork belly spectrum appears to be less broadband due to the frequency dependent attenuation from propagation through the tissue.

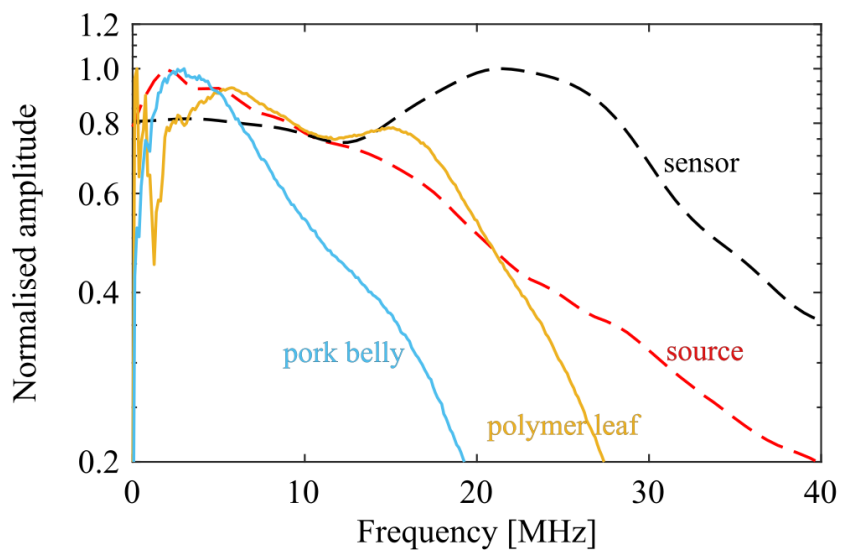

Fig. 14. Average frequency spectra of received data from polymer leaf skeleton phantom (yellow) and pork belly tissue sample (blue); also shown are frequency spectra of the FP sensor (dashed black) and the optical ultrasound source (dashed red). 


\section{ACKNOWLEDGMENT}

The authors would like to thank Michael Brown and James Guggenheim, University College London (UCL) for useful discussions and Olivia Wright (UCL) for technical support.

\section{REFERENCES}

[1] S.-L. Chen, "Review of laser-generated ultrasound transmitters and their applications to all-optical ultrasound transducers and imaging," Appl. Sci., vol. 7, no. 1, p. 25, 2016.

[2] S. Noimark et al., "Polydimethylsiloxane composites for optical ultrasound generation and multimodality imaging," Adv. Funct. Mater., vol. 28, no. 9, pp. 1-16, 2018.

[3] S. Rajagopal, T. Sainsbury, B. E. Treeby, and B. T. Cox, "Laser generated ultrasound sources using carbon-polymer nanocomposites for high frequency metrology," J. Acoust. Soc. Am., vol. 144, no. 2, pp. 584-597, 2018.

[4] P. Kruizinga, B. Cox, N. De Jong, P. Beard, A. F. W. Van Der Steen, and G. Van Soest, "Plane wave ultrasound imaging with a broadband photoacoustic source," IEEE Int. Ultrason. Symp., pp. 1414-1416, 2012.

[5] D. Thompson, D. Gasteau, and S. Manohar, "Spatially compounded plane wave imaging using a laser-induced ultrasound source," Photoacoustics, vol. 18, no. 100154, 2020.

[6] Y. Hou, J. S. Kim, H. Sheng-Wen, S. Ashkenazi, L. J. Guo, and M. O'Donnell, "Characterization of a broadband all-optical ultrasound transducer - from optical and acoustical properties to imaging," IEEE Trans. Ultrason. Ferroelectr. Freq. Control, vol. 55, no. 8, pp. $1867-$ $1877,2008$.

[7] C. Sheaff and S. Ashkenazi, "A polyimide - etalon thin film structure for all-optical high-frequency ultrasound transduction," IEEE Trans. Ultrason. Ferroelectr. Freq. Control, vol. 59, no. 10, pp. 2254-2261, 2012.

[8] S. Noimark et al., "Carbon-nanotube-PDMS composite coatings on optical fibers for all-optical ultrasound imaging," Adv. Funct. Mater., vol. 26, no. 46, pp. 8390-8396, Dec. 2016.

[9] R. J. Colchester, E. Z. Zhang, C. A. Mosse, P. C. Beard, I Papakonstantinou, and A. E. Desjardins, "Broadband miniature optical ultrasound probe for high resolution vascular tissue imaging," Biomed. Opt. Express, vol. 6, no. 4, pp. 1502-1511, 2015.

[10] E. Zhang, J. Laufer, and P. Beard, "Backward-mode multiwavelength photoacoustic scanner using a planar Fabry-Perot polymer film ultrasound sensor for high resolution three-dimensional imaging of biological tissues," Appl. Opt., vol. 47, no. 4, pp. 561-577, 2008.

[11] J. Laufer et al., "In vivo preclinical photoacoustic imaging of tumor vasculature development and therapy.," J. Biomed. Opt., vol. 17, no. 5, p. 056016, 2012.

[12] A. P. Jathoul et al., "Deep in vivo photoacoustic imaging of mammalian tissues using a tyrosinase-based genetic reporter," Nat. Photonics, vol. 9, pp. 239-246, 2015.

[13] E. J. Alles et al., "Acoustical characterisation of carbon nanotubeloaded polydimethylsiloxane used for optical ultrasound generation," IEEE Int. Ultrason. Symp. Proc., 2017.

[14] W. F. Beach, "Xylylene Polymers," in Encyclopedia of Polymer Science and Technology. Hoboken, NJ, USA: Wiley, 2004.

[15] B. T. Cox and P. C. Beard, "The frequency-dependent directivity of a planar Fabry-Perot polymer film ultrasound sensor," IEEE Trans. Ultrason. Ferroelectr. Freq. Control, vol. 54, no. 2, pp. 394-404, 2007.

[16] S. J. Norton and M. Linzer, "Ultrasonic reflectivity imaging in three dimensions: Exact inverse scattering solutions for plane, cylindrical, and spherical apertures," IEEE Trans. Biomed. Eng., vol. BME-28, no. 2, pp. 202-220, 1981.

[17] J. Cheng and J. Lu, "Extended high-frame rate imaging method with limited-diffraction beams," IEEE Trans. Ultrason. Ferroelectr. Freq. Control, vol. 53, no. 5, pp. 880-899, 2006.

[18] E. Martin, E. Z. Zhang, J. A. Guggenheim, P. C. Beard, and B. E. Treeby, "Rapid spatial mapping of focused ultrasound fields using a planar Fabry-Pérot sensor," IEEE Trans. Ultrason. Ferroelectr. Freq. Control, vol. 64, no. 11, pp. 1711-1722, 2017.

[19] J. A. Guggenheim, E. Z. Zhang, and P. C. Beard, "A method for measuring the directional response of ultrasound receivers in the range $0.3 \mathrm{MHz}$ to $80 \mathrm{MHz}$ using a laser generated ultrasound source,"
IEEE Trans. Ultrason. Ferroelectr. Freq. Control, vol. 64, no. 12, pp. 1857-1863, 2017.

[20] E. Maneas et al., "Gel wax-based tissue-mimicking phantoms for multispectral photoacoustic imaging," Biomed. Opt. Express, vol. 9, no. 3, pp. 1151-1163, 2018.

[21] V. L. Newhouse, N. M. Bilgutay, J. Saniie, and E. S. Furgason, "Flaw-to-grain echo enhancement by split-spectrum processing," Ultrasonics, vol. March, pp. 59-68, 1982.

[22] T. J. Allen et al., "High pulse energy fibre laser as an excitation source for photoacoustic tomography," Opt. Express, vol. 28, no. 23, pp. 34255-34265, 2020.

[23] M. Tanter and M. Fink, "Ultrafast imaging in biomedical ultrasound," IEEE Trans. Ultrason. Ferroelectr. Freq. Control, vol. 61, no. 1, pp. 102-119, 2014.

[24] G. Montaldo, M. Tanter, J. Bercoff, N. Benech, and M. Fink, "Coherent plane-wave compounding for very high frame rate ultrasonography and transient elastography," IEEE Trans. Ultrason. Ferroelectr. Freq. Control, vol. 56, no. 3, pp. 489-506, 2009.

[25] R. K. Ing, F. Gires, and M. Fink, "Focusing and beamsteering of laser generated ultrasound," in IEEE Ultrasonics Symposium, 1989, pp. $539-544$.

[26] N. Huynh, O. Ogunlade, E. Zhang, B. Cox, and P. Beard, "Photoacoustic imaging using an 8-beam Fabry-Perot scanner," in Proceedings of SPIE, 2016, vol. 9708, Art. no. 97082L.

[27] T. J. Allen, E. Zhang, and P. C. Beard, "Fully parallelised read-out of a Fabry-Perot ultrasound sensor using an InGaAs camera for fast photoacoustic imaging (Conference Presentation)," in Proceedings of SPIE, 2020, vol. 11240, Art. no. $112400 \mathrm{U}$.

[28] R. Ansari, E. Z. Zhang, A. E. Desjardins, and P. C. Beard, "Alloptical forward-viewing photoacoustic probe for high-resolution 3D endoscopy," Light Sci. Appl., vol. 7, no. 75, 2018.

[29] E. J. Alles, N. Fook Sheung, S. Noimark, E. Z. Zhang, P. C. Beard, and A. E. Desjardins, "A reconfigurable all-optical ultrasound transducer array for 3D endoscopic imaging," Sci. Rep., vol. 7, no. 1, pp. 1-9, 2017. 